\title{
Production of 4-ethylphenol from lignin depolymerization in novel surfactant-free
}

\section{microemulsion reactor}

Juanhua Kong, ${ }^{\text {a }}$ Lixia Li, ${ }^{\text {a }}$ Qiang Zeng, ${ }^{\text {a Jinxing Long, }}{ }^{\text {a Hongyan He}},{ }^{\mathrm{b}}$ Yingying Wang, ${ }^{\mathrm{c}}$ Sijie $\mathrm{Liu}^{\square \mathrm{a}}$ and Xuehui $\mathrm{Li}^{\square \mathrm{a}}$

a School of Chemistry and Chemical Engineering, State Key Laboratory of Pulp and Paper Engineering, South China University of technology, Guangzhou, 510640, China.

${ }^{b}$ Beijing Key Laboratory of Ionic Liquids Clean Process, Institute of Process Engineering, Chinese Academy of Sciences, Beijing, 100190, China.

c South China Advanced Institute for Soft Matter Science and Technology, South China University of Technology, Guangzhou, 510640, China.

*To whom correspondence should be addressed. e-mail address: cexhli@scut.edu.cn (Li, X.H.); cesjliu@scut.edu.cn (Liu, S.J.) 


\section{Figures and tables}

Figure S1. Quantitative ${ }^{31} \mathrm{P}$ NMR spectra of lignins with 2-chloro-1, 3, 2-dioxaphospholane using cyclohexanol as the IS substance.

Figure S2. $\lambda_{\max }$ values of $\mathrm{MO}$ in the microemulsions at $\mathrm{R}_{\mathrm{P} / \mathrm{O}}=9.05$ with different $\mathrm{W}_{\mathrm{W}}$ value and different solvents.

Figure S3. FT-IR spectra diagram of different solvents with $\mathrm{R}_{\mathrm{P} / \mathrm{O}}=9.05$ and the fitted curve O-H stretching band.

Figure S4. UV-vis spectra of 4-nitroaniline for different ionic liquids in ethanol.

Figure S5. GC-MS diagram of volatile products from lignin depolymerization in microemulsion.

Table S1. The average molecular weight of raw lignins.

Table S2. Different hydroxyl groups in lignin as determined by quantitative ${ }^{31} \mathrm{P}$ NMR.

Table S3. Effect of the microemulsion composition on lignin depolymerization.

Table S4. Hammett functions and $\mathrm{pH}$ values of ionic liquids.

Table S5. Different lignins depolymerization in SFME reactor.

Table S6. The quantitative data of volatile products from lignin depolymerization in microemulsion. 


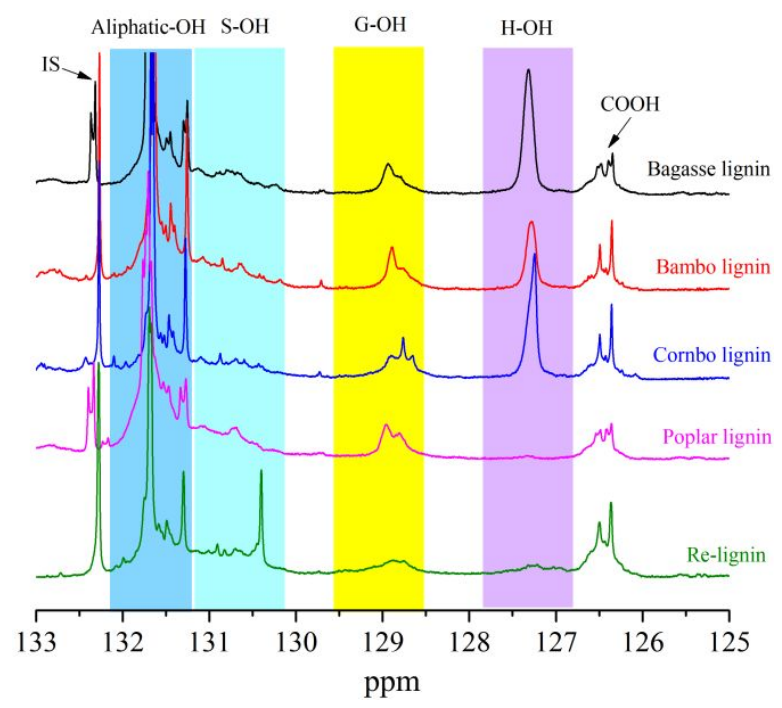

Figure S1. Quantitative ${ }^{31} \mathrm{P}$ NMR spectra of lignins with 2-chloro-1, 3, 2dioxaphospholane using cyclohexanol as the IS substance.
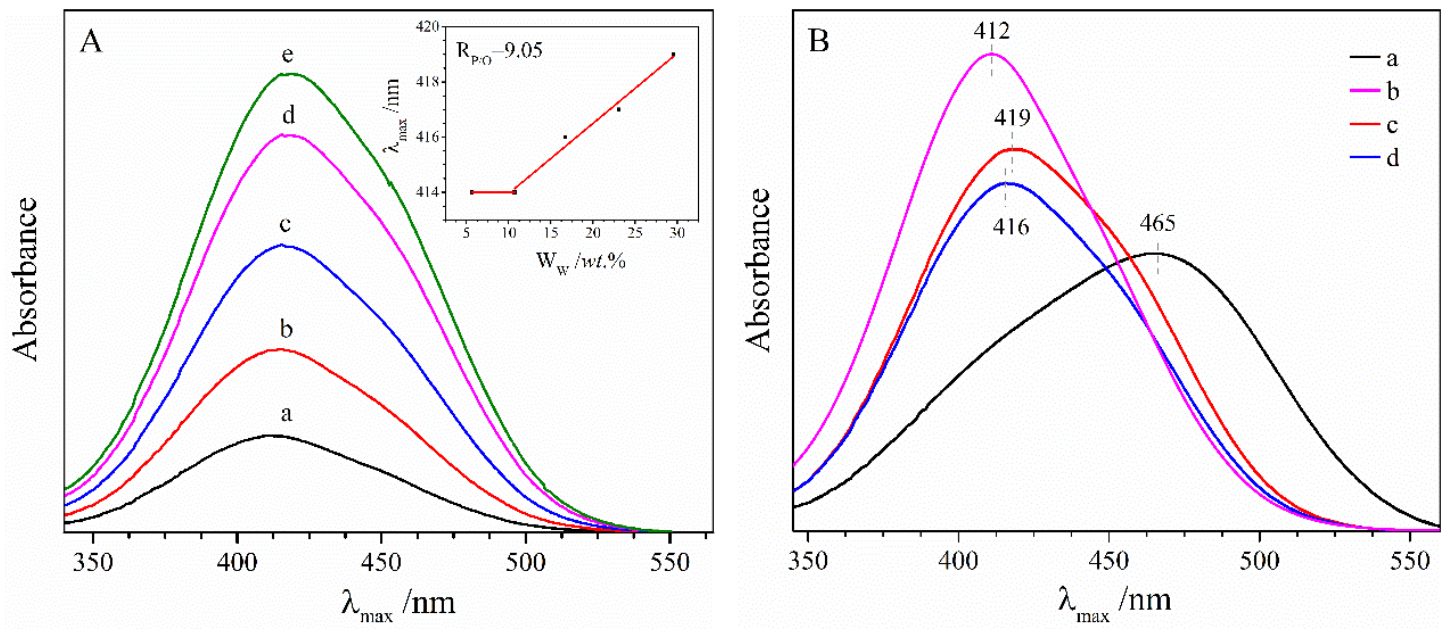

Figure S2. $\lambda_{\max }$ values of $\mathrm{MO}$ in (A) different microemulsions at $\mathrm{R}_{\mathrm{P} / \mathrm{O}}=9.05$ with the $\mathrm{W}_{\mathrm{W}}$ of (a) 5.6, (b) 10.7, (c) 16.7, (d) 23.1 and (e) 29.6, and (B) different solvents (a) water, (b) 2propanol, (c) a binary system with $\mathrm{R}_{\mathrm{P} / \mathrm{W}}=4.50, \mathrm{Wo}=0 w t . \%$, and (d) a microemulsion with

$$
\mathrm{R}_{\mathrm{P} / \mathrm{W}}=4.50, \mathrm{Wo}_{\mathrm{o}}=8.3 w t . \% \text {. }
$$



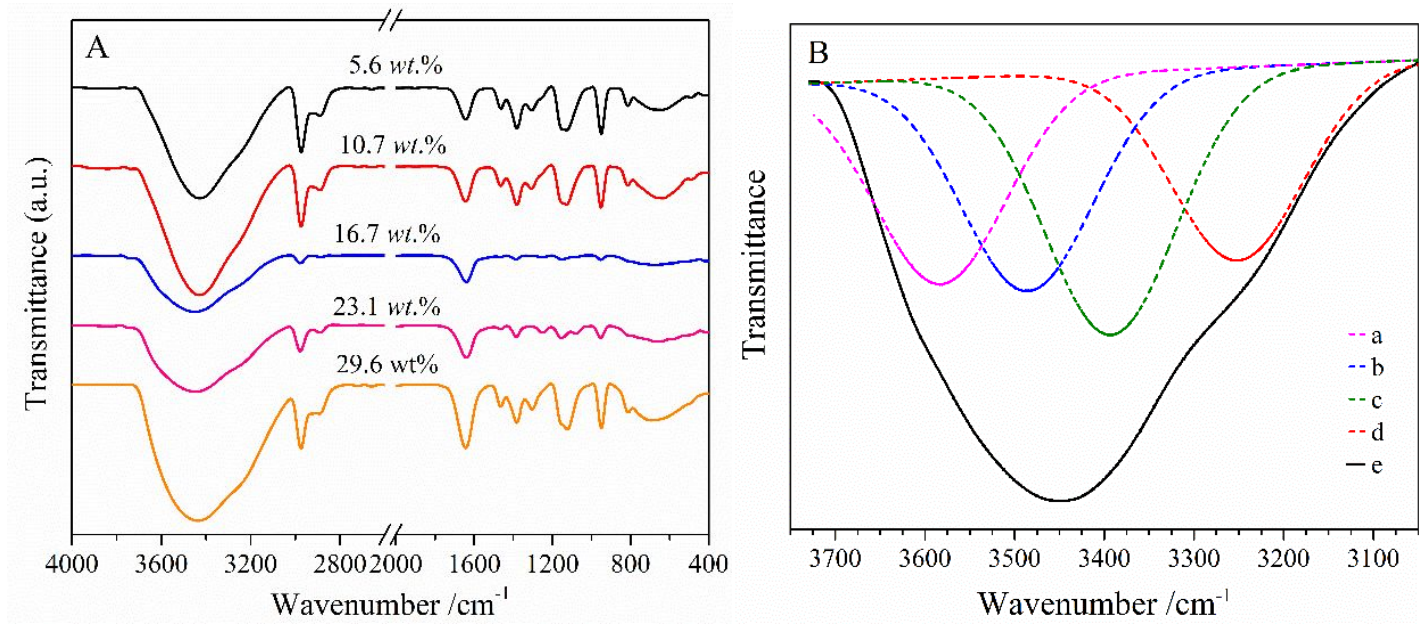

Figure S3. FT-IR spectra diagram of (A) different solvents with $\mathrm{R}_{\mathrm{P} / \mathrm{O}}=9.05$ and (B) the fitted curve O-H stretching band of (a) trapped water, (b) bound water, (c) 2-propanol, (d) free water and e the experimental one in the microemulsion with $\mathrm{W}_{\mathrm{W}}=16.7 \mathrm{wt} . \%$. 


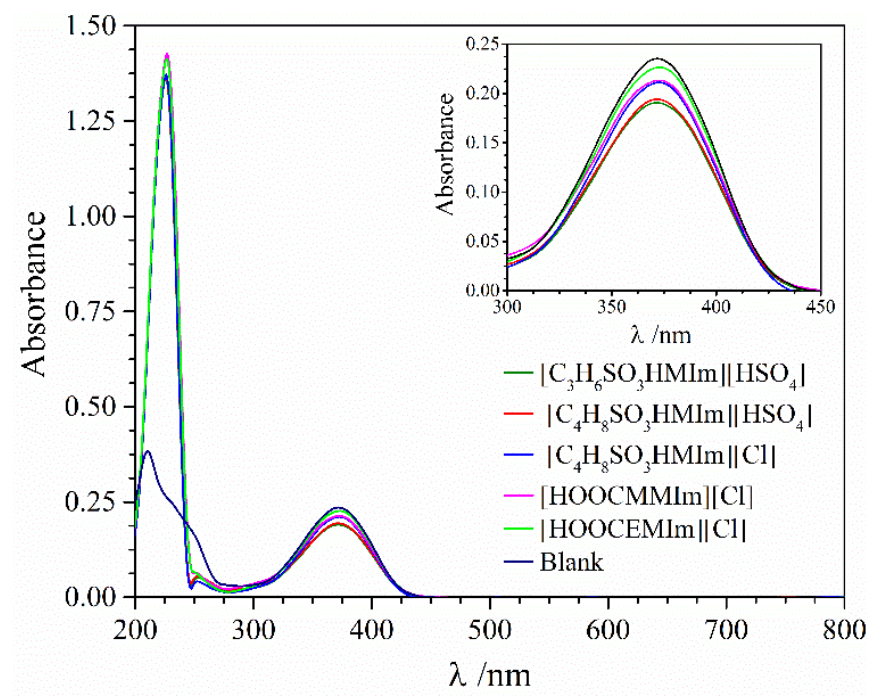

Figure S4. UV-vis spectra of 4-nitroaniline for different ionic liquids in ethanol.

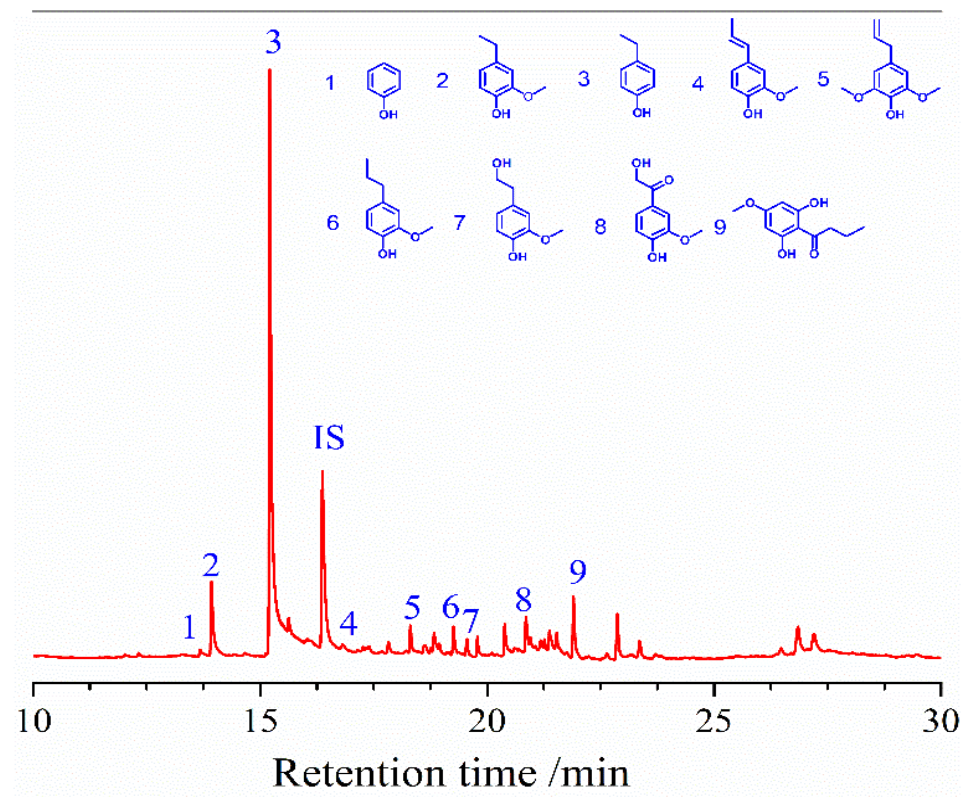

Figure S5. GC-MS diagram of volatile products from lignin depolymerization in microemulsion. Note: IS-internal standard. 
Table S1. The average molecular weight of raw lignins.

\begin{tabular}{ccccc}
\hline Name & $\mathrm{M}_{\mathrm{n}}$ & $\mathrm{M}_{\mathrm{w}}$ & $\mathrm{M}_{\mathrm{z}}$ & $\mathrm{D}$ \\
\hline Bagasse lignin & 1404.7 & 3523.5 & 8301.3 & 2.51 \\
Bamboo lignin & 1153.8 & 3943.1 & 10920 & 3.42 \\
Corncob lignin & 1134.3 & 3295.8 & 7593.1 & 2.91 \\
Poplar lignin & 1258.2 & 3155.9 & 5704.6 & 2.51 \\
\hline
\end{tabular}

Note: $M_{n}$-number average molecular weight; $M_{w}$-weight average molecular weight; $M_{z}$-Z-average molecular weight; $D=M_{w} / M_{n}$, dispersion degree.

Table S2. Different hydroxyl groups in lignin as determined by quantitative ${ }^{31} \mathrm{P}$ NMR.

\begin{tabular}{|c|c|c|c|c|c|c|c|}
\hline \multirow{2}{*}{ Name } & \multirow{2}{*}{$\begin{array}{l}\text { Aliphatic-OH } \\
\qquad / \mathrm{mmol} \mathrm{g}^{-1}\end{array}$} & \multicolumn{4}{|c|}{ Phenolic-OH $/ \mathrm{mmol} \mathrm{g}^{-1}$} & \multirow{2}{*}{$\begin{array}{l}\text { Acids } \mathrm{COOH} \\
/ \mathrm{mmol} \mathrm{g}^{-1}\end{array}$} & \multirow{2}{*}{$\begin{array}{c}\text { Total } \\
/ \mathrm{mmol} \mathrm{g}^{-1}\end{array}$} \\
\hline & & S & $\mathrm{G}$ & $\mathrm{H}$ & S:G:H & & \\
\hline $\begin{array}{l}\text { Bagasse } \\
\text { lignin }\end{array}$ & 2.66 & 0.47 & 0.40 & 1.06 & $24.4: 20.7: 54.9$ & 0.47 & 5.06 \\
\hline $\begin{array}{c}\text { Bamboo } \\
\text { lignin }\end{array}$ & 1.54 & 0.77 & 0.32 & 0.33 & $54.2: 22.5: 23.2$ & 0.27 & 3.23 \\
\hline $\begin{array}{c}\text { Corncob } \\
\text { lignin }\end{array}$ & 1.66 & 0.31 & 0.46 & 0.82 & $19.5: 28.9: 51.6$ & 0.47 & 3.72 \\
\hline $\begin{array}{l}\text { Poplar } \\
\text { lignin }\end{array}$ & 3.67 & 0.32 & 0.47 & 0.01 & $40.0: 58.8: 1.2$ & 0.44 & 4.91 \\
\hline Re-lignin & 2.51 & 0.70 & 0.34 & 0.09 & $62.0: 30.1: 8.0$ & 0.88 & 3.64 \\
\hline
\end{tabular}


Table S3. Effect of the microemulsion composition on lignin depolymerization.

\begin{tabular}{|c|c|c|c|c|c|c|c|c|c|}
\hline \multirow[b]{2}{*}{ Entry } & \multicolumn{3}{|c|}{ System $/ w t . \%$} & \multirow{2}{*}{$\begin{array}{l}\text { SFME } \\
\text { type }\end{array}$} & \multirow{2}{*}{$\begin{array}{l}\text { Solubility } \\
\qquad / \mathrm{g} \mathrm{L}^{-1}\end{array}$} & \multicolumn{4}{|c|}{ Yield $/ \mathrm{mg} \mathrm{g}^{-1}$} \\
\hline & Water & 2-Propanol & $n$-Octane & & & $Y_{E P}$ & $\mathrm{Y}_{\text {Others }}$ & $\mathrm{Y}_{\text {Total }}$ & $\begin{array}{l}\mathrm{S}_{\mathrm{EP}} \\
/ \%\end{array}$ \\
\hline 1 & 0 & 0 & 100 & - & & - & - & - & \\
\hline 2 & 100 & 0 & 0 & - & & $1.7 \pm 0.3$ & $3.7 \pm 1.7$ & $5.4 \pm 1.6$ & 31.5 \\
\hline 3 & 0 & 100 & 0 & - & 7.0 & $7.4 \pm 0.7$ & $\begin{array}{c}43.4 \pm 3 \\
6\end{array}$ & $\begin{array}{c}50.8 \pm 3 . \\
1\end{array}$ & 14.6 \\
\hline 4 & 0 & 50 & 50 & - & 2.3 & $5.3 \pm 0.3$ & $\begin{array}{c}51.9 \pm 3 . \\
0\end{array}$ & $\begin{array}{c}57.2 \pm 3 . \\
0\end{array}$ & 9.3 \\
\hline 5 & 50 & 50 & 0 & - & 37.0 & $\begin{array}{c}44.1 \pm 3 . \\
9\end{array}$ & $\begin{array}{c}16.1 \pm 1 \\
7\end{array}$ & $\begin{array}{c}60.2 \pm 4 \\
4\end{array}$ & 73.2 \\
\hline 6 & 33.3 & 33.4 & 33.3 & - & & $\begin{array}{c}47.3 \pm 3 \\
5\end{array}$ & $\begin{array}{c}19.4 \pm 2 . \\
2\end{array}$ & $\begin{array}{c}66.7 \pm 2 . \\
8\end{array}$ & 70.9 \\
\hline 7 & 25.0 & 50.0 & 25.0 & & & $\begin{array}{c}48.8 \pm 2 . \\
0\end{array}$ & $\begin{array}{c}30.9 \pm 4 . \\
4\end{array}$ & $\begin{array}{c}79.7 \pm 3 . \\
4\end{array}$ & 61.2 \\
\hline 8 & 0 & 90.5 & 9.5 & - & 9.4 & $\begin{array}{c}12.4 \pm 2 . \\
2\end{array}$ & $\begin{array}{c}10.6 \pm 2 . \\
7\end{array}$ & $\begin{array}{c}23.0 \pm 3 . \\
5\end{array}$ & 53.9 \\
\hline 9 & 5.6 & 85.0 & 9.4 & B.C & 16.8 & $\begin{array}{c}37.4 \pm 2 . \\
2\end{array}$ & $\begin{array}{c}43.4 \pm 3 . \\
4\end{array}$ & $\begin{array}{c}80.8 \pm 2 . \\
3\end{array}$ & 46.3 \\
\hline 10 & 10.7 & 80.4 & 8.9 & $\mathrm{O} / \mathrm{W}$ & 28.1 & $\begin{array}{c}58.5 \pm 3 . \\
7\end{array}$ & $\begin{array}{c}29.2 \pm 3 \\
2\end{array}$ & $\begin{array}{c}87.7 \pm 2 . \\
8\end{array}$ & 66.7 \\
\hline 11 & 16.7 & 75.0 & 8.3 & $\mathrm{O} / \mathrm{W}$ & 59.2 & $\begin{array}{c}63.4 \pm 4 . \\
0\end{array}$ & $\begin{array}{c}30.1 \pm 4 . \\
4\end{array}$ & $\begin{array}{c}93.5 \pm 4 . \\
4\end{array}$ & 67.8 \\
\hline 12 & 23.1 & 69.3 & 7.6 & $\mathrm{O} / \mathrm{W}$ & 64.8 & $\begin{array}{c}54.8 \pm 3 . \\
6\end{array}$ & $\begin{array}{c}26.5 \pm 2 . \\
9\end{array}$ & $\begin{array}{c}81.3 \pm 4 . \\
2\end{array}$ & 67.4 \\
\hline 13 & 29.6 & 63.4 & 7.0 & $\mathrm{O} / \mathrm{W}$ & 64.9 & $\begin{array}{c}53.4 \pm 4 . \\
2\end{array}$ & $\begin{array}{c}22.4 \pm 2 \\
4\end{array}$ & $\begin{array}{c}75.8 \pm 3 . \\
7\end{array}$ & 70.4 \\
\hline 14 & 39.4 & 54.6 & 6.0 & - & & $\begin{array}{c}47.5 \pm 2 . \\
\quad 6\end{array}$ & $\begin{array}{c}18.4 \pm 1 . \\
2\end{array}$ & $\begin{array}{c}65.9 \pm 1 . \\
7\end{array}$ & 72.1 \\
\hline $15^{\mathrm{a}}$ & 16.7 & 75.0 & 8.3 & $\mathrm{O} / \mathrm{W}$ & 59.2 & - & $\begin{array}{c}40.1 \pm 3 . \\
0\end{array}$ & $\begin{array}{c}40.1 \pm 3 . \\
0\end{array}$ & - \\
\hline 16 & 18.2 & 81.8 & 0 & - & 43.4 & $\begin{array}{c}42.0 \pm 2 \\
4\end{array}$ & $\begin{array}{c}25.2 \pm 2 \\
1\end{array}$ & $\begin{array}{c}67.2 \pm 3 \\
4\end{array}$ & 62.5 \\
\hline 17 & 17.7 & 79.4 & 2.9 & $\mathrm{O} / \mathrm{W}$ & 47.9 & $\begin{array}{c}51.5 \pm 2 . \\
6\end{array}$ & $\begin{array}{c}26.4 \pm 2 . \\
6\end{array}$ & $\begin{array}{c}77.9 \pm 3 . \\
8\end{array}$ & 66.1 \\
\hline 18 & 15.8 & 71.0 & 13.2 & B.C & 26.8 & $\begin{array}{c}57.3 \pm 3 . \\
1\end{array}$ & $\begin{array}{c}25.8 \pm 1 \\
6\end{array}$ & $\begin{array}{c}83.1 \pm 1 . \\
8\end{array}$ & 69.0 \\
\hline 19 & 15.0 & 67.5 & 17.5 & B.C & 21.8 & $\begin{array}{c}54.7 \pm 3 . \\
2\end{array}$ & $\begin{array}{c}32.1 \pm 2 . \\
0\end{array}$ & $\begin{array}{c}86.8 \pm 2 . \\
7\end{array}$ & 63.0 \\
\hline 20 & 13.7 & 61.7 & 24.6 & $\mathrm{~W} / \mathrm{O}$ & 14.1 & $\begin{array}{c}49.8 \pm 3 . \\
8\end{array}$ & $\begin{array}{c}22.0 \pm 2 . \\
8\end{array}$ & $\begin{array}{c}71.8 \pm 3 . \\
0\end{array}$ & 69.4 \\
\hline
\end{tabular}




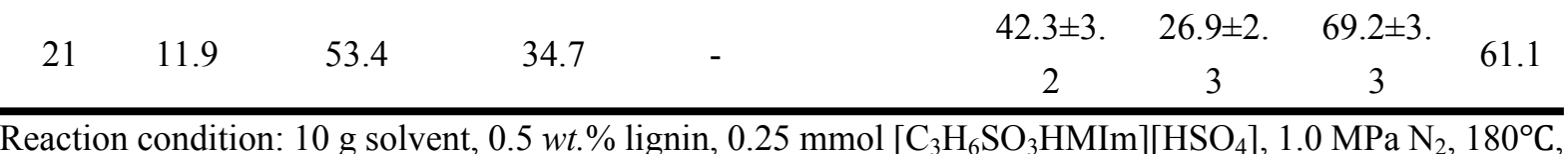

4 h. Note: a- no catalyst, EP represents the product of 4-ethylphenol, $S_{E P}$ is the selectivity of EP in phenolic monomers. 
Table S4. Hammett functions and $\mathrm{pH}$ values of ionic liquids.

\begin{tabular}{ccccccccc}
\hline & & & & & & \multicolumn{3}{c}{$\mathrm{pH}$ value } \\
\cline { 6 - 8 } Entry & Catalyst & $\mathrm{A}_{\max }$ & {$[\mathrm{I}] / \%$} & {$\left[\mathrm{IH}^{+}\right] / \%$} & $\mathrm{H}_{\mathrm{o}}$ & $\mathrm{C}_{1}$ & $\mathrm{C}_{2}$ \\
& & & & & & & $(2.5$ & $(2.5$ \\
& & & & & & & $\mathrm{mM})$ & $\mathrm{mM})$ \\
\hline 1 & $\mathrm{Blank}$ & & & & & & $/$ & $/$ \\
2 & $\mathrm{H}_{2} \mathrm{SO}_{4}$ & & & & $/$ & 1.51 & 2.40 \\
3 & {$\left[\mathrm{C}_{3} \mathrm{H}_{6} \mathrm{SO}_{3} \mathrm{HMIm}_{[}\right]\left[\mathrm{HSO}_{4}\right]$} & 0.192 & 81.7 & 18.3 & 1.64 & 1.51 & 2.38 \\
4 & {$\left[\mathrm{C}_{4} \mathrm{H}_{8} \mathrm{SO}_{3} \mathrm{HMIm}\right]\left[\mathrm{HSO} \mathrm{H}_{4}\right]$} & 0.196 & 83.4 & 16.6 & 1.69 & 1.51 & 2.37 \\
5 & {$\left[\mathrm{C}_{4} \mathrm{H}_{8} \mathrm{SO}_{3} \mathrm{HMIm}\right][\mathrm{Cl}]$} & 0.209 & 88.9 & 11.1 & 1.90 & 2.27 & 3.27 \\
6 & {$[\mathrm{HOOCMMIm}][\mathrm{Cl}]$} & 0.213 & 90.6 & 9.4 & 1.98 & 1.97 & 2.71 \\
7 & {$[\mathrm{HOOCEMIm}][\mathrm{Cl}]$} & 0.226 & 96.2 & 3.8 & 2.39 & 2.62 & 3.17 \\
\hline
\end{tabular}

$\mathrm{H}_{\mathrm{O}}=\mathrm{pKa}+\log [\mathrm{I}] /\left[\mathrm{IH}^{+}\right]$; indicator: 4-nitroaniline $(\mathrm{pKa}=0.99)$. Note: the alcohol solution containing $25.0 \mathrm{mM}$ ionic liquid and $2.0 \mathrm{mg} \mathrm{L}^{-1}$ 4-nitroaniline for Hammett functions determination; ionic liquid in aqueous solution for $\mathrm{pH}$ value test.

Table S5. Different lignins depolymerization in SFME reactor.

\begin{tabular}{|c|c|c|c|c|c|}
\hline \multirow{2}{*}{\multicolumn{2}{|c|}{ Feedstock }} & \multicolumn{3}{|c|}{ Yield $/ \mathrm{mg} \mathrm{g}^{-1}$} & \multirow{2}{*}{$\begin{array}{c}\text { Mass balance } \\
\qquad \%\end{array}$} \\
\hline & & $Y_{E P}$ & $Y_{\text {Others }}$ & $\mathrm{Y}_{\text {Total }}$ & \\
\hline \multirow{4}{*}{$\begin{array}{l}\text { Organosolv } \\
\text { lignin }\end{array}$} & bamboo lignin & $16.3 \pm 2.2$ & $22.7 \pm 2.7$ & $39.0 \pm 1.4$ & 88.7 \\
\hline & bagasse lignin & $63.4 \pm 4.1$ & $30.1 \pm 4.2$ & $93.5 \pm 5.0$ & 92.5 \\
\hline & corncob lignin & $75.7 \pm 3.5$ & $52.4 \pm 5.8$ & $128.1 \pm 5.0$ & 92.9 \\
\hline & poplar lignin & $6.8 \pm 1.5$ & $13.0 \pm 1.2$ & $19.8 \pm 0.9$ & 94.4 \\
\hline \multirow{3}{*}{$\begin{array}{l}\text { Technical } \\
\text { lignin }\end{array}$} & $\begin{array}{c}\text { sodium } \\
\text { lignosulphonate }\end{array}$ & - & $12.6 \pm 2.5$ & $12.6 \pm 2.5$ & 88.4 \\
\hline & alkali lignin & - & $15.4 \pm 3.1$ & $15.4 \pm 3.1$ & 96.0 \\
\hline & deakali lignin & - & $6.2 \pm 0.8$ & $6.2 \pm 0.8$ & 93.9 \\
\hline
\end{tabular}

Reaction condition: $10 \mathrm{~g}$ microemulsion $\left(\mathrm{W}_{\mathrm{W}}: \mathrm{W}_{\mathrm{P}}: \mathrm{W}_{\mathrm{O}}=16.7: 75.0: 8.3, w t . \%\right), 0.5 w t . \%$ lignin, $0.25 \mathrm{mmol}$ $\left[\mathrm{C}_{3} \mathrm{H}_{6} \mathrm{SO}_{3} \mathrm{HMIm}\right]\left[\mathrm{HSO}_{4}\right], 1.0 \mathrm{MPa} \mathrm{N}{ }_{2}, 180^{\circ} \mathrm{C}, 4 \mathrm{~h}$. 
Table S6. The quantitative data of volatile products from lignin depolymerization in microemulsion.

\begin{tabular}{|c|c|c|c|c|}
\hline $\begin{array}{l}\mathrm{RT} \\
/ \mathrm{min}\end{array}$ & Name & $\begin{array}{l}\text { Chemical } \\
\text { Formula }\end{array}$ & $\begin{array}{l}\text { Product } \\
\text { type }\end{array}$ & $\begin{array}{l}\text { Yield } \\
/ \mathrm{mg} \cdot \mathrm{g}^{-1}\end{array}$ \\
\hline $\begin{array}{c}13.7 \\
0\end{array}$ & phenol & $\mathrm{C}_{6} \mathrm{H}_{6} \mathrm{O}$ & $\mathrm{H}$ & 0.9 \\
\hline $\begin{array}{c}13.9 \\
5\end{array}$ & 4-ethyl-2-methoxy-phenol & $\mathrm{C}_{9} \mathrm{H}_{12} \mathrm{O}_{2}$ & FA, G & 11.4 \\
\hline $\begin{array}{c}15.2 \\
4\end{array}$ & 4-ethylphenol & $\mathrm{C}_{8} \mathrm{H}_{10} \mathrm{O}$ & $p \mathrm{CA}, \mathrm{H}$ & 105.6 \\
\hline $\begin{array}{c}16.8 \\
2\end{array}$ & 2-methoxy-4-propenyl-phenol & $\mathrm{C}_{10} \mathrm{H}_{12} \mathrm{O}_{2}$ & G & 2.4 \\
\hline $\begin{array}{c}18.3 \\
3\end{array}$ & 4-ally-2,6-dimethoxy-phenol & $\mathrm{C}_{11} \mathrm{H}_{14} \mathrm{O}_{3}$ & $\mathrm{~S}$ & 3.7 \\
\hline $\begin{array}{c}18.8 \\
5\end{array}$ & 2-methoxy-4-propyl-phenol & $\mathrm{C}_{10} \mathrm{H}_{14} \mathrm{O}_{2}$ & G & 2.8 \\
\hline $\begin{array}{c}19.2 \\
8\end{array}$ & 4-(2-hydroxy-ethyl)-2-methoxy-phenol & $\mathrm{C}_{9} \mathrm{H}_{12} \mathrm{O}_{3}$ & G & 2.8 \\
\hline $\begin{array}{c}20.4 \\
1\end{array}$ & $\begin{array}{l}\text { 2-hydroxy-1-(4-hydroxy-3-methoxy-phenyl)- } \\
\text { ethanone }\end{array}$ & $\mathrm{C}_{9} \mathrm{H}_{10} \mathrm{O}_{4}$ & G & 3.0 \\
\hline $\begin{array}{c}21.9 \\
4\end{array}$ & 1-(2,6-dihydroxy-4-methoxy-phenyl)-butan-1-one & $\mathrm{C}_{11} \mathrm{H}_{14} \mathrm{O}_{4}$ & - & 6.1 \\
\hline
\end{tabular}

Reaction condition: $10 \mathrm{~g}$ microemulsion $\left(\mathrm{W}_{\mathrm{W}}: \mathrm{W}_{\mathrm{P}}: \mathrm{W}_{\mathrm{O}}=16.7: 75.0: 8.3, w t . \%\right), 0.5$ wt.\% lignin, $0.25 \mathrm{mmol}$ $\left[\mathrm{C}_{3} \mathrm{H}_{6} \mathrm{SO}_{3} \mathrm{HMIm}\right]\left[\mathrm{HSO}_{4}\right], 1.0 \mathrm{MPa} \mathrm{N}, 180^{\circ} \mathrm{C}$. Note: $\mathrm{H}, \mathrm{G}$ and $\mathrm{S}$ represent the product from the structural units of the $p$-coumaryl alcohol (H), coniferyl alcohol (G), and sinapyl alcohol (S) in lignin respectively.

\section{References}

(1) Akim, L. G.; Argyropoulos, D. S.; Jouanin, L.; Leplé, J. C.; Pilate, G.; Pollet, B.; Lapierre, C. Quantitative ${ }^{31} \mathrm{P}$ NMR spectroscopy of lignins from transgenic poplars. Holzforschung, 2001, 55, 386-390.

(2) Balakshin, M.; Capanema, E. On the quantification of lignin hydroxyl groups with ${ }^{31} \mathrm{P}$ and ${ }^{13} \mathrm{C}$ NMR spectroscopy. J. Wood Chem. Technol., 2015, 35, 220-237. 